\title{
画HAD
}

ISSN-L: 2530-5115

(c) (1) (2)

DOI: http://doi.org/10.22585/hospdomic.v2i2.44

\section{Infografías en las ciencias de la salud: aplicación al cuidado domiciliario}

Infographics in health sciences: application to home care

\author{
María Sanz-Lorente',2, Ramón Castejón-Bolea' \\ 1. Universidad Miguel Hernández, Departamento de Salud Pública e Historia de la Ciencia, Campus San Joan \\ d'Alacant, Alicante, España. \\ 2. Conselleria de Sanidad Universal y Salud Pública, Centro de Salud Pública de Manises, Manises, España.
}

Correspondencia/Correspondence

María Sanz-Lorente

Universidad Miguel Hernández, Departamento

de Salud Pública e Historia de la Ciencia,

Campus San Joan d'Alacant, Alicante, España.

msanzlor@gmail.com

Recibido/Received

27.02.2018

Aceptado/Accepted

20.03.2018

CÓMO CITAR ESTE TRABAJO | HOW TO CITE THIS PAPER

Sanz-Lorente M, Castejón-Bolea R. Infografías en las ciencias de la salud: aplicación al cuidado domiciliario. Hosp Domic. 20 18;2(2):67-78 


\section{RESUMEN}

La infografía es una técnica que combina imágenes y textos creada con el fin de comunicar información de manera visual para facilitar su transmisión. Es una herramienta efectiva para el aprendizaje en ciencias de la salud, siendo el proceso del diseño de la misma lo que resulta más beneficioso desde el punto de vista docente.

La visualización de la información, mediante infografías, es un factor clave en la popularización de los aspectos técnico-biomédicos.

En el área de la salud la infografía se ha convertido en un recurso importante para explicar procedimientos de atención, enfermedades, procesos médicos, funcionamiento de órganos y sistemas o situaciones relacionadas con la salud. En consecuencia, muchas instituciones sanitarias utilizan ya esta herramienta para informar a la población en general.

No se pudo recuperar modelos de infografía sobre usos o aplicaciones en los servicios de atención de salud a domicilio. Sin embargo, si se encontraron al realizar la búsqueda en Google Images ${ }^{\circledR}$ y en plataformas como Pinterest ${ }^{\circledR}$, la mayoría sobre beneficios de la atención domiciliaria, cuidados o dirigidas a personas mayores. Por tanto, serían necesarios estudios que evidenciaran la validez de las infografías en el cuidado del enfermo domiciliario.

Sería deseable que los profesionales relacionados con los servicios de atención de salud a domicilio se involucraran más en el desarrollo de nuevas herramientas, como las infografías, que facilitaran la comunicación entre ellos y las personas necesitadas de sus cuidados.

Palabras clave: Servicios de Atención de Salud a Domicilio; Comunicación en Salud; Compresión de Datos; Infografía; Promoción de la Salud; Educación en Salud.

\section{ABSTRACT}

Infographic is a combination of images and texts, in order to communicate information visually to facilitate its transmission. It is an effective tool for the learning of health science, being the design process of this which becomes more beneficial from the teaching point of view.

The display of information, through infographics, is a key factor in the popularization of technicalbiomedical aspects.

In the health area infographics has become an important resource to explain care procedures, diseases, medical processes, functioning of organs and systems, or health-related situations. As a result, many health institutions already use this tool to inform the population in general.

Unfortunately, it was not retrieved scientific literature on uses or applications of infographics in home health care services. However, it was found when performing the search in Google Images $₫$ or platforms as Pinterest $®$, the most on benefits of home care, care or aimed to elderly people. There would therefore be necessary studies that show the validity of infographics in the care of patients at home.

It would be desirable that health professionals related to health-care services to home were more involved in the development of new tools, such as infographics that facilitate the communication between them and the needy of their care.

Keywords: Home Care Services; Health Communication; Data Compression; Infographic; Health Promotion; Health Education. 


\section{ANTECEDENTES}

La infografía (del inglés, infographics = informational graphics), es una combinación de textos y de imágenes sintéticas, explicativas y fáciles de entender, con el fin de comunicar información de manera visual para facilitar su transmisión. Como ejemplo, se podría hacer referencia al mapa de un tesoro o a un esquema del sistema solar.

Así, según Serra (1), las principales características de una infografía serían:

- Visual - Aunque se apoya, o acentúa algunas de las imágenes, con la palabra.

- Sin voluntad estética - El estilo gráfico de las imágenes no debe dificultar la comprensión del tema tratado.

- Las imágenes responden a la necesidad de comunicar algo - Cada elemento incluido en una infografía debe responder a una necesidad informativa (evitar distraer la atención, de quien lo lea, con imágenes cuyo único objetivo sea ilustrar).

- Información cierta y precisa - Deben responder a criterios de claridad, síntesis y veracidad.

En un infografía la composición de imagen y palabra es indivisible, de tal modo que una no se entiende en ausencia de la otra, siendo el resultado mucho más preciso que por separado; donde no llega la imagen lo hace la palabra y viceversa.

Si bien, la Real Academia Española da una definición vana e intrascendente de los que es infografía, "técnica de elaboración de imágenes mediante ordenador», estas representaciones gráficas tienen una existencia que data de muchísimos años atrás. Su eclosión se produce en el Renacimiento (siglos $\mathrm{XV}$ y XVI), mediante las representaciones realizadas a mano de mapas, cartas de navegación o funcionamiento de máquinas. Es necesario, en este apartado, resaltar la figura de Leonardo Da Vinci que representó "infográficamente" sus ideas, inventos y máquinas; ver figura 1.

Ahora bien, si hay un hito destacable, sería el mapa del metro de Londres que Henry Beck diseñó, durante su tiempo libre, en 1931 y que se convirtió en uno de los diseños gráficos más influyentes de la humanidad. A partir de este mapa se han creado la gran mayoría, por no decir la totalidad, de los planos de transporte público de las ciudades de todo el mundo. Su gran visión consistió en obviar las relaciones de distancia reales entre las estaciones y la utilización identificativa del color para los diferentes ramales (2).

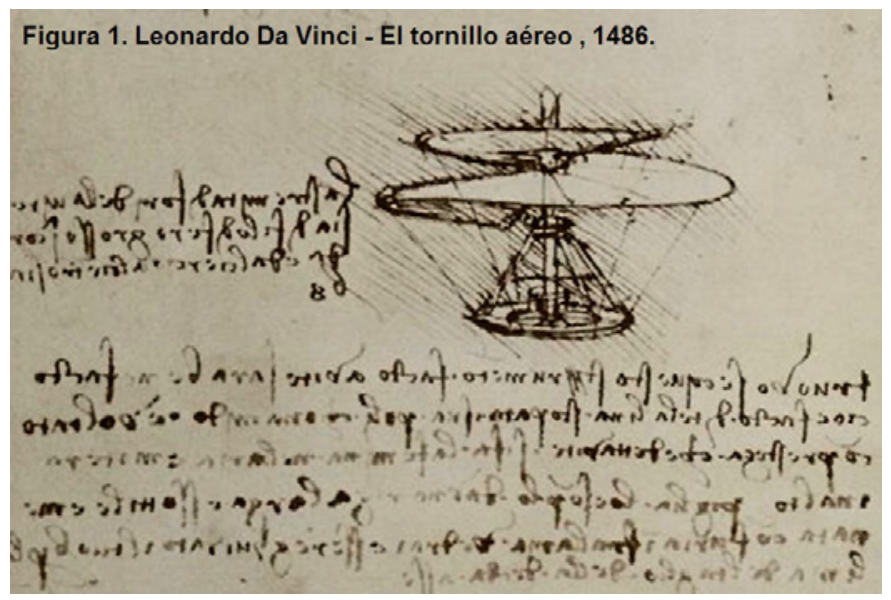


En la actualidad, debemos ser concientes que se está inmerso en el mundo de la imagen, la cual ha desplazado en gran medida otras formas habituales de comunicación, si bien convive y se interrelaciona con ellas. Un buen cuadro gráfico debe ser sencillo, completo, ético, bien diseñado y adecuado con la información que presenta. El más apropiado será aquel que ayude mejor a quien lo lea y que combine de una forma óptima con su contenido. Por tanto, la infografía debe ser, simplemente, una herramienta que armonice utilidad con visualidad, con el fin último de comprender la información (3); ver figura 2.

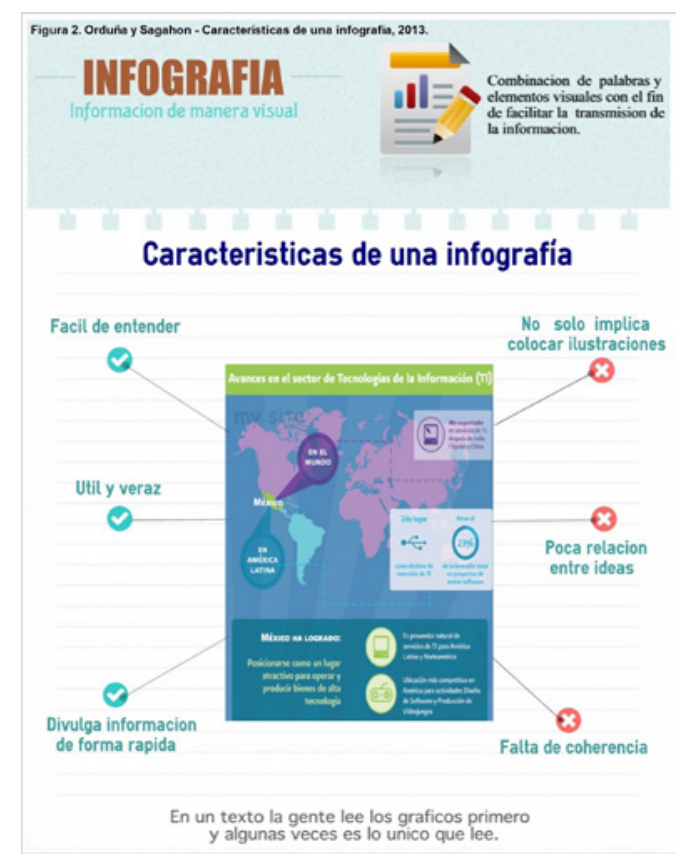

La conclusión que se puede obtener, en línea con lo expuesto por Serra (1), «es que la infografía debe informar de forma sencilla, pero no simple y que la edición del contenido no debe ceñirse únicamente a la velocidad de lectura. En ocasiones es necesario ofrecer más información al lector sacrificando la inmediatez. Además, en tanto que herramienta visual, buena parte de una infografía es estética, no obstante esta debe mantenerse supeditada a la ética. Son dos caras de una misma moneda. La estética carece de valor sin la ética».

\section{USOS DE LA INFOGRAFÍA EN EDUCACIÓN}

La relación entre comunicación y educación, en la sociedad de la información, constituye, sin duda, uno de los campos decisivos de la transformación de la cultura y de la educación en nuestros días, y una de las áreas en las que realmente la humanidad se juega su auténtico progreso, o retroceso, en los años venideros (4).

Como señala, acertadamente, Reinhardt (5), «no se pretende demostar que un recurso específico, como podría ser una infografía, es la solución absoluta y única para el problema pedagógico 
en cuestión: sólo se trata de indagar en las posibilidades didácticas que este recurso ofrece y ver en qué situaciones su intervención aportaría beneficios significativos en el proceso de enseñanzaaprendizaje»

El uso de la infografía como material de enseñanza tiene la misión de transmitir noticias, eventos, o datos, de una forma visual, facilitando la comprensión de determinada información compleja o poco familiar, al estimular el interés del lector. Convirtiéndose, por tanto, en un recurso educativo (6).

La infografía didáctica conforma una oportunidad de investigar de forma sistematizada y fundamentada, a la vez que promueve el desarrollo de la escritura y comunicación visual, a la hora de elaborarla. Al mismo tiempo, según Guzmán-Cedillo et al. (7), «el contenido presentado en las infografías didácticas permite: informar y aprender. Pese a estas características, son pocos los estudiantes (y docentes) que en su discurso se apropian de la infografía como herramienta de aprendizaje cuando refieren que pueden utilizarla para otros momentos en su formación o como documento para compartir temas de salud con más personas».

Estos antecedentes indican que la infografía tiene el potencial de promover la comprensión e interacción con la información en el proceso de aprendizaje, pues a través de imágenes y textos se puede impulsar la asociación de ideas, así como incrementar el conocimiento sobre los temas tratados.

Dentro de las estrategias de enseñanza las infografías son una excelente herramienta para abordar contenidos, siendo tremendamente útiles en la docencia de las ciencias de la salud (anatomía, microbiología, botánica, etc.). Es evidente que diferentes ámbitos de estudio, y la medicina es un claro ejemplo de ello, han utilizado la imagen a la hora de plantear y trasmitir el conocimiento (8). A ello, indudablemente, ha contribuido el avance de las tecnologías de la información y la comunicación (TIC); ver figura 3.

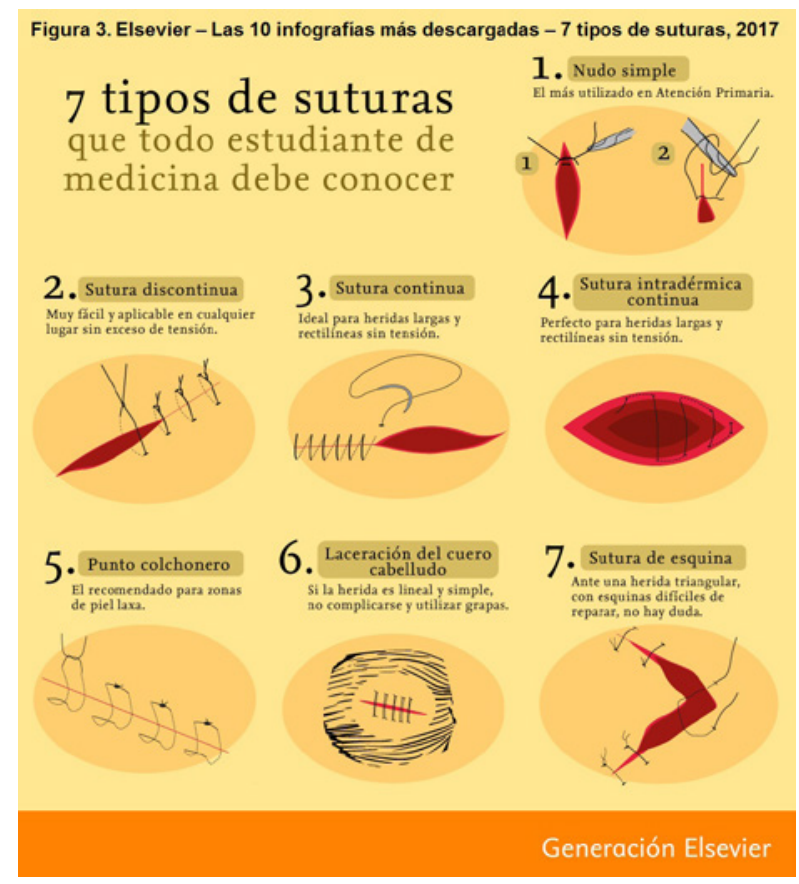


Shanks et al. (9), comprobaron recientemente que la infografía facilitaba el aprendizaje de los estudiantes sobre cuestiones de ciencias de la salud y que resultaba un recurso efectivo para la enseñanza. Por su parte, la investigación de Scott et al. (10), concluyó que si bien las personas pueden formarse una opinión del contenido de una infografía en pocos segundos, es el proceso del diseño de la misma lo que resulta más beneficioso desde el punto de vista docente.

Los resultados de un estudio, realizado por Alcíbar en 2017 (11), sugirieron que la visualización de la información, mediante infografías, fue un factor clave en la popularización de los aspectos técnico-biomédicos de la última epidemia del virus del Ébola, además de contribuir activamente a construir, en palabras del autor, una narración de su naturaleza.

Aguirre et al. (12), refirieron que «de forma general la secuencia al elaborar infografías didácticas consiste en la elección de un tema, su investigación, la organización, el bosquejo, el diseño, la revisión de la infografía y su publicación». Habría que reflexionar sobre la pertinencia de incrementar pasos o actividades como la retroalimentación (como parte de una autoevaluación y coevaluación), además de tener la oportunidad de presentar la infografía a la audiencia para la que se creó (7).

Se podría afirmar que las infografías son textos expositivos cuya finalidad es ampliar el conocimiento del receptor sobre algún tema. Su emisor, por ello, es siempre alguien con un conocimiento superior sobre el contenido que se desarrolla.

Para Muñoz García (13), «son más sintéticas que los vídeos, más narrativas que un esquema, más atractivas que las tablas de datos, más exploratorias que las presentaciones tradicionales y, a diferencia de los textos escritos, permiten visualizar la información que presentan, pudiendose asimilar sus contenidos con "un golpe de vista". El empleo de recursos tecnológicos en el aula es un condicionante que capta la atención del alumnado e incrementa de manera positiva el interés de éstos por las materias impartidas y un seguimiento más activo de las clases. A pesar de la asiduidad de las TIC dentro y fuera del aula, el poder visual de las infografías ha conseguido impresionar al alumnado, así como facilitar la adquisición, repaso o ampliación de contenidos, lo cual se considera trascendental».

Por último, y no menos importante, hay que tener en cuenta que la vista constituye una de las vías de entrada más importantes a través de las cuales la persona accede al conocimiento (14). En consecuencia, la lectura de una infografía debe seguir el orden lógico utilizado por el público diana (en el mundo occidental: de izquierda a derecha y de arriba abajo), apoyando con números o letras el orden de lectura y destacando, cuando sea necesario, el elemento que tiene mayor valor informativo.

\section{LA INFOGRAFÍA EN LAS CIENCIAS DE LA SALUD}

La salud es uno de los temas que más interesa a la población española, lo cual ha llevado a los medios de comunicación a dar más cabida a este tema: en diez años se han duplicado los artículos sobre salud en la prensa. Como sugirió González-Pacanowski et al. (15), «el gran interés que la población tiene en estos temas, representa una oportunidad única para que los actores implicados directamente en ese área fomenten, gracias al uso de las nuevas herramientas tecnológicas, distintas iniciativas que tengan como objetivo la educación de la población en hábitos de salud».

La capacidad de precisión y síntesis ha vinculado cutural e históricamente a la infografía con la ciencia. Pero, al ser "solo" una herramienta, por si misma no define a quien sirve. Necesita un adjetivo que la acompañe y que defina lo verdaderamente importante: el tipo de información que esta obligada a contener (1). 
A día de hoy, la infografía logra enfrentar al lector a un tema particular cuyo tratamiento le invita a descubrir nuevos datos que, en ocasiones, se actualizan a medida que se desarrollan (16).

Buljan et al. (17), en 2017, realizaron un estudio, a partir de una revisión sistemática Cochrane, para comprobar la utilidad de la infografía en la trasmisión del conocimiento sobre información de salud (conocimiento sobre la información de salud presentada, experiencia de lectura y facilidad de uso percibida), a poblaciones profesionales y no profesionales, teniendo como elemento de comparación el resumen científico y un resumen en lenguaje llano. El resultado que encontraron fue que no se apreció diferencias en relación con el conocimiento percibido entre los 3 modos de presentar la información, pero los participantes (profesionales y no profesionales) prefirieron la infografía y le dieron calificaciones más altas para la experiencia de lectura y la facilidad de uso. Estos resultados avalan que se publiquen infografías, incluso en revistas científicas, para facilitar el intercambio y la comunicación del conocimiento $(18,19)$. También, para apoyar a los profesionales de la salud en la implementación de recomendaciones y promover el cambio de comportamiento de una población diana (20). Infografías que muestran de forma clara, amena y gráfica, importantes temas de la salud pública y el cuidado de la salud; una forma accesible y atractiva de describir y explicar estos temas.

La infografía se ha convertido en una estrategia para educar al paciente que aporta beneficios como la inmediatez, acompañada de la optimización del tiempo, la didáctica y la ética. A la vez que mantiene el rigor científico al que deben someterse todas las informaciones emitidas por los actores del sector salud. También, al aprovechar su formato digital, se difunde a través de distintos medios $(7,15)$.

En el área de la salud, la elaboración de infografías se ha convertido en un recurso importante para explicar procedimientos de atención, enfermedades, procesos médicos, funcionamiento de órganos y sistemas o situaciones relacionadas con la salud, con el objetivo de dar claridad a las personas sobre los tratamientos y enfermedades. Muchas instituciones sanitarias utilizan ya esta herramienta para informar a la población en general. Algunos ejemplos pueden consultarse en las siguientes direcciones electrónicas:

- World Health Organization: https://goo.gl/g3mq9z

- Centers for Disease Control and Prevention: https://goo.gl/hbqR57

- Instituto Nacional de Salud Pública de México: https://goo.gl/9Da2jy

Queda patente, que el carácter didáctico y educativo de la infografía la convierte en una de las actividades de información online más usadas en el sector salud. Teniendo en cuenta que las campañas de comunicación de salud pueden influir en el comportamiento sanitario de la población (21), que la población está muy acostumbrada a la cultura de la imagen (22), y que en la actualidad hay una fusión de soportes tecnológicos, parece claro que la difusión de infografías por parte de las organizaciones implicadas en la salud es una de las estrategias más claras para lograr ese doble objetivo de divulgación científica rigurosa y de educación a la población (15).

Por todo ello, González-Pacanowski et al. (15), apuntaron que en la edición de infografías en el área de la salud se deben seguir tres reglas principales:

- Cumplir el rigor informativo, facilitando la comprensión de acontecimientos.

- Respetar el rigor científico.

- Ser consciente de la labor didáctica de su trabajo: su objetivo será aumentar el nivel de formación del receptor.

A estas tres reglas es necesario añadir que el autor también debe respetar la ética, la cual, debe estar presente en todas las actividades de comunicación y salud $(1,23)$.

En definitiva, la infografía es un tipo de comunicación, con un alto grado de apoyo visual, que presenta mensajes concretos para facilitar la comprensión de un tema complejo con igual o su- 
perior validez que otros medios textuales de divulgación (7). McCrorie et al. (24), observaron que «una comprensión firme de la información de salud mejora la capacidad en la toma decisiones de los pacientes y puede mejorar la relación con los profesionales sanitarios. La infografía también se debería utilizar a nivel de la población para transmitir mensajes de salud pública».

\section{APLICACIÓN AL CUIDADO DOMICILIARIO}

El cuidado domiciliario constituye hoy una modalidad de atención que permite solventar las dificultades derivadas de la sobreocupación hospitalaria y la cronicidad, los cuales constituyen un problema de interés en los países desarrollados y que pueden ser manejados en el domicilio del paciente como una opción costo-efectiva y segura. Para ello, es necesario buscar estrategias que permitan su desarrollo, gestión de riesgos y modelos de atención, logrando mejorar las condiciones de salud de la población (25).

En el domicilio, paciente y familia pasan de espectadores a protagonistas, cambia su actitud, asumen una posición más activa tanto en la comunicación como en la toma de decisiones y asumen más responsabilidad y más participación en los cuidados (26).

El cuidado tiene entre sus pilares el establecimiento de una buena comunicación con el enfermo, mediada principalmente por el lenguaje verbal. Sin embargo, se sabe que no solo la palabra es mediación en la comunicación. Además, los mensajes pueden ser olvidados, recordados o modificados de acuerdo con el significado que le dan los pacientes según su comprensión o utilidad (27). Por tanto, siempre será aconsejable utilizar herramientas comunicativas que permitan recordar e interpretar las instrucciones de forma correcta.

En este sentido, la infografía hace realidad el hecho de transmitir gráficamente y de forma organizada los conceptos relacionados con una idea, producto o servicio, evitando al usuario invertir el poco tiempo del que dispone en arduas lecturas y posiblemente malas o erróneas interpretaciones, y además facilita la rápida asimilación del mensaje.

Una búsqueda realizada en las principales bases de datos bibliográficas sobre ciencias de la salud (MEDLINE, Embase, Scopus, PsycINFO y Web of Science) y en las colecciones de revistas ScienceDirect y SciELO, no recuperó literatura científica sobre usos o aplicaciones de la infografía en los servicios de atención de salud a domicilio. Sin embargo, sí se encontraron al realizar la búsqueda en Google Images ${ }^{\oplus}$ (https://images.google.com/) y en plataformas como Pinterest ${ }^{\circledast}$ (https:// www.pinterest.es/), la mayoría sobre beneficios de la atención domiciliaria (figura 4), cuidados (figura 5) o, sobre todo, dirigidas a personas mayores (figura 6).

De todos modos, hay que dejar constancia que existe un estudio donde se sugiere que las infografías en Pinterest contienen pocos elementos sobre comportamiento adecuado en salud (28) y que, según Brigham (29), el aumento de las fuentes de datos gratuitas alojadas en Internet y la necesidad de contenidos por parte de la población ha llevado a la popularización de las infografías, con claros beneficios pero también con potenciales problemas, y que en la Web 2.0 hay que tener muy en cuenta la validez de la información aunque provenga de "fuentes confiables" (30). 


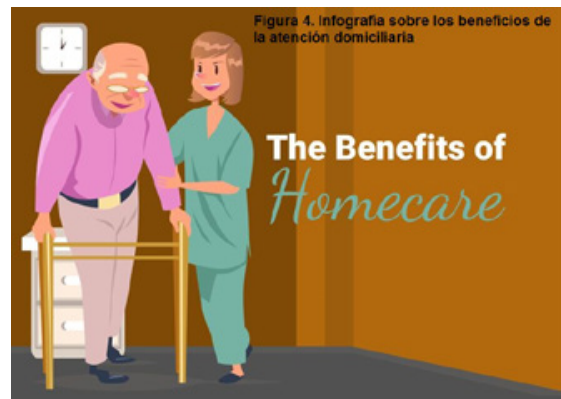

Recover faster at home

Be in a familiar environment

Be surrounded by family and friends

Less-expensive than facilities

Home care patients have better health outcomes

Receive help for light housekeeping tasks

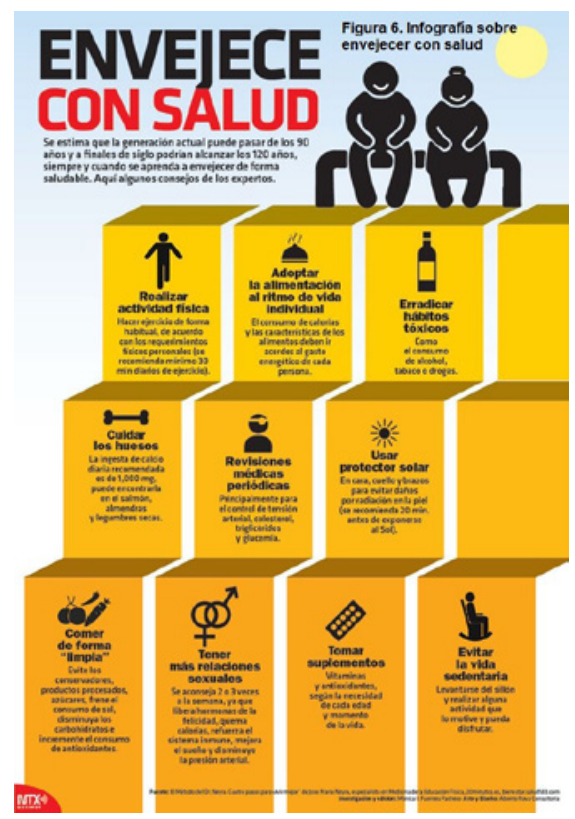

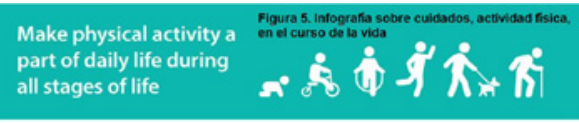

\begin{tabular}{|c|c|c|}
\hline $\begin{array}{c}6 \text { OUT OF } 10 \\
\text { Whakhtathen }\end{array}$ & 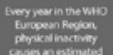 & 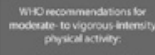 \\
\hline 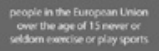 & $\begin{array}{l}1 \text { MLLLION } \\
\text { DEATHS }\end{array}$ & $2^{150} \div$ \\
\hline
\end{tabular}

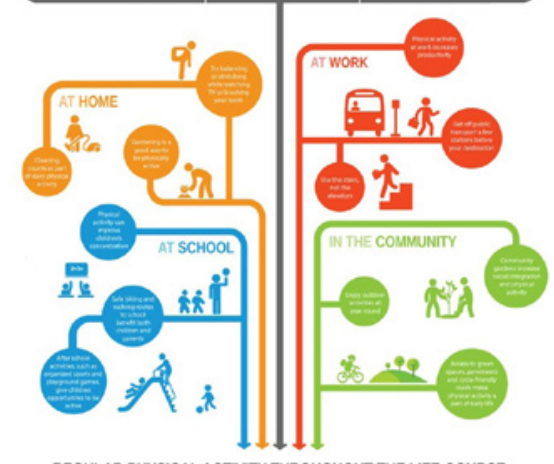

REGULAR PHYSICAL ACTIVIYY THROUGHOUT THE LIFE-COURSE ENABLES PEOPLE TO LIVE BETTER AND LONGER LIVES 


\section{CONCLUSIONES}

La infografía es una herramienta efectiva para el aprendizaje de las ciencias de la salud, siendo el proceso del diseño de la misma lo que resulta más beneficioso desde el punto de vista docente.

La visualización de la información, mediante infografías, fue un factor clave en la popularización de los aspectos técnico-biomédicos.

En el área de la salud la infografía se ha convertido en un recurso importante para explicar procedimientos de atención, enfermedades, procesos médicos, funcionamiento de órganos y sistemas o situaciones relacionadas con la salud. En consecuencia, muchas instituciones sanitarias utilizan ya esta herramienta para informar a la población en general.

No se localizó información, en las bases de datos bibliográficas tradicionales, al buscar sobre usos o aplicaciones de la infografía en los servicios de atención de salud a domicilio. Sin embargo, si se encontró al realizar la búsqueda en Google Images ${ }^{\circledR}$ o en plataformas como Pinterest ${ }^{\circledR}$, la mayoría sobre beneficios de la atención domiciliaria, cuidados o dirigidas a personas mayores. Por tanto, serían necesarios estudios que evidenciaran la validez de las infografías en el cuidado del enfermo domiciliario.

Sería deseable que los profesionales de la salud relacionados con los servicios de atención de salud a domicilio se involucraran más en el desarrollo de nuevas herramientas, como las infografías, que facilitaran la comunicación entre ellos y las personas necesitadas de sus cuidados.

\section{BIBLIOGRAFÍA}

1. Serra J. No hay estética sin ética [monografía en Internet]. Santa Coloma de Cervelló, España: Scribd Inc; 2017 [citado 25 de enero de 2018]. Disponible en: https://goo.gl/n62kby

2. Raizman D. History of modern design: graphics and products since the Industrial Revolution. London, United Kingdom: Laurence King Publishing Ltd; 2003.

3. Valero Sancho JL. La infografía: Técnicas, análisis y usos periodísticos. Barcelona, España: Publicaciones de la Universidad Autónoma de Barcelona; 2001.

4. Pérez Tornero JM. Comunicación y educación en la sociedad de la información: nuevos lenguajes y conciencia crítica. Barcelona, España: Editorial Paidós; 2000.

5. Reinhardt NV. Infografía didáctica: Producción interdisciplinaria de infografías para la diversidad cultural. Cuad Cent Estud Diseño Comun - Ens. 2010;10(31):119-91.

6. Brigas J, Gonçalves J, Milheiro S. Infographics in the education context. En: Sousa Reis C, Tadeu P, Paira T, editores. Proceedings Book for the Conference on Enabling Teachers for Entrepreneurship Education. Guarda, Portugal: Guarda Polytechnic Institute; 2013. p. 51-5.

7. Guzmán-Cedillo Y, Lima-Villeda N, Ferreira-Rosa S. La experiencia de elaborar infografías didácticas sobre diversidad sexual. Rev Lat Comun Soc. 2015;(70):961-81.

8. Falk NL. Infographic Development by Accelerated Bachelor of Science in Nursing Students: An Innovative Technology-Based Approach to Public Health Education. Nurs Educ Perspect. 2016;37(5):299-301. DOI: 10.1097/01.NEP.0000000000000066; PMID: 27740570

9. Shanks JD, Izumi B, Sun C, Martin A, Byker Shanks C. Teaching undergraduate students to visualize and communicate public health data with infographics. Front Public Health. 2017;5:315. DOI: 10.3389/fpubh.2017.00315; PMID: 29226120 
10. Scott $\mathrm{H}$, Fawkner S, Oliver CW, Murray A. How to make an engaging infographic? Br J Sports Med. 2017;51(16):1183-4. DOI: 10.1136/bjsports-2016-097023; PMID: 28039124

11. Alcíbar M. Information visualisation as a resource for popularising the technical-biomedical aspects of the last Ebola virus epidemic: The case of the Spanish reference press. Public Underst Sci. 2017;963662517702047. DOI: 10.1177/0963662517702047; PMID: 28393588

12. Aguirre CR, Menjívar Valencia E, Morales HL. Elaboración de infografías: hacia el desarrollo de competencias del siglo XXI. Diá-Logos. 2015;(15):23. DOI: 10.5377/dialogos.v0i15.2207

13. Muñoz García E. Uso didáctico de las infografías. Espiral - Cuad Profr. 2014;7(14):37-43.

14. Valero-Sancho JL. Visualidad del producto gráfico. Rev Lat Comun Soc [revista en Internet]. 2002;51 [citado 25 de enero de 2018]. Disponible en: https://goo.gl/PyZxVJ

15. González-Pacanowski T, Medina P. Comunicación online en el sector salud: Valor de la infografía. El Prof Inf. 2009;18(4):413-20. DOI: 10.3145/epi.2009.jul.08

16. Marín Ochoa BE. La infografía digital, una nueva forma de comunicación [tesis doctoral]. BarceIona, España: Universidad Autónoma de Barcelona; 2009.

17. Buljan I, Malički M, Wager E, Puljak L, Hren D, Kellie F, et al. No difference in knowledge obtained from infographic or plain language summary of a Cochrane systematic review: three randomized controlled trials. J Clin Epidemiol. 2017; pii: S0895-4356(17)30490-0. DOI: 10.1016/j. jclinepi.2017.12.003; PMID: 29269021

18. Infographic: world of waste. Science. 2012;337(6095):664-7. DOI: 10.1126/science.337.6095.664; PMID: 22879498

19. Reid H, Foster C. Infographic. Physical activity benefits for adults and older adults. Br J Sports Med. 2017;51(19):1441-2. DOI: 10.1136/bjsports-2016-096811; PMID: 27581161

20. Smith R, Shakespeare J, Williams Z, Knight M, Foster C. Physical activity for pregnant women: an infographic for healthcare professionals. Br J Gen Pract J R Coll Gen Pract. 2017;67(663):460. DOI: 10.3399/bjgp17X692801; PMID: 28963415

21. Hornik RC. Public health communication: evidence for behaviour change. Mahwah, USA: Lawrence Erlbaum Associates; 2002.

22. Manovich L. El lenguaje en los nuevos medios de comunicación: la imagen en la era digital. Barcelona, España: Editorial Paidós; 2005.

23. Guttman N. Ethics in health communication interventions. En: Thompson TL, Dorsey A, Parrott R, Miller K, editores. Handbook of health communication. Mahwah, USA: Lawrence Erlbaum Associates; 2003. p. 651-79.

24. McCrorie AD, Donnelly C, McGlade KJ. Infographics: Healthcare Communication for the Digital Age. Ulster Med J. 2016;85(2):71-5. PMID: 27601757

25. Sánchez M, Fuentes G. Gestion clínica de programas de cuidado domiciliario. Rev CES Salud Pública. 2016;7(2):1-11.

26. Gallud Romero J. Por qué sigo creyendo en el hospital a domicilio. Hosp Domic. 2017;1(2):5764. DOI: 10.22585/hospdomic.v1i2.16

27. Madrigal Ramírez MC, Forero Pulido C, Escobar CL. La comunicación, piedra angular en el cuidado de enfermería. Investig Enferm Imagen Desarro. 2013;15(2):49-63.

28. Wilkinson JL, Strickling K, Payne HE, Jensen KC, West JH. Evaluation of Diet-Related Infographics on Pinterest for Use of Behavior Change Theories: A Content Analysis. JMIR MHealth UHealth. 2016;4(4):e133. DOI: 10.2196/mhealth.6367; PMID: 27932316 
Artículo Especial: Sanz-Lorente, Castejón-Bolea

29. Brigham TJ. Feast for the Eyes: An Introduction to Data Visualization. Med Ref Serv Q. 2016;35(2):215-23. DOI: 10.1080/02763869.2016.1152146; PMID: 27054537

30. Sanz-Lorente M, Guardiola-Wanden-Berghe R. Evaluación de la calidad de las páginas Web sobre el Hospital a Domicilio: el Indicador de Credibilidad como factor pronóstico. Hosp Domic. 2017; 1(2):73-82. DOI: 10.22585/hospdomic.v1i2.11 